\title{
Paciente con displasia ventricular derecha arritmogénica: alteraciones electrocardiográficas, tratamiento e indicaciones de los programas de rehabilitación cardiaca
}

\author{
Concepción Cuenca Gonzáleza ${ }^{a}$, Isabel Flores Torres ${ }^{b}$, Ana María Herranz Torrubiano ${ }^{c}$ \\ y María Fernanda Berzal Cantalejod.
}

\begin{abstract}
a Especialista en Medicina Familiar y Comunitaria. Especialista en Medicina Física y Rehabilitación. Servicio de Medicina Física y Rehabilitación. Hospital Clínico de San Carlos. Madrid (España).

${ }^{b}$ Especialista en Medicina Física y Rehabilitación. Servicio de Medicina Física y Rehabilitación. Hospital La Inmaculada. Huércal-Overa. Almería (España).

c Especialista en Medicina Familiar y Comunitaria. Centro de Salud La Alameda de Osuna. Madrid (España).

d Especialista en Medicina Familiar y Comunitaria. Especialista en Anatomía Patológica. Servicio de Anatomía Patológica. Hospital General Río Carrión. Palencia (España).
\end{abstract}

Correspondencia: Concepción Cuenca González. Avda. Martín Lagos, s/n. C.P. 28045 - Madrid (España).

Correo electrónico:

cuchacuenca@gmail.com.

Recibido el 24 de septiembre de 2014.

Aceptado para su publicación el 27 de octubre de 2014 .

\begin{abstract}
RESUMEN
La displasia ventricular derecha arritmogénica es una de las causas más comunes de muerte súbita en adultos jóvenes. La clínica es variable y va desde pacientes asintomáticos, arritmias ventriculares y supraventriculares, insuficiencia cardíaca derecha o biventricular, hasta muerte súbita, en cualquier fase de la enfermedad. Desde las consultas de Atención Primaria podemos captar a pacientes asintomáticos con sospecha de presentar esta patología. No existen directrices claras con respecto al tratamiento de pacientes asintomáticos y sin arritmias. En pacientes con riesgo moderado es aconsejable el tratamiento farmacológico. En los pacientes, con riesgo elevado para desarrollar arritmias, se debe considerar la ablación por radiofrecuencia, la colocación de un Desfibrilador Automático Implantable (DAl) y el trasplante cardiaco. Existe evidencia de los indudables beneficios que a nivel físico y psicológico consiguen los Programas de Rehabilitación Cardiaca en portadores de DAl, y de los escasos riesgos del entrenamiento bien programado.
\end{abstract}

Palabras Clave. Displasia Ventricular Derecha Arritmogénica. Tratamiento. Rehabilitación.

\begin{abstract}
Patient with arrhythmogenic right ventricular dysplasia: electrocardiographic abnormalities, treatment and indications for cardiac rehabilitation programs

The arrhythmogenic right ventricular dysplasia is one of the most common causes of sudden death in young adults. The clinical profile is variable, ranging from asymptomatic patients, ventricular and supraventricular arrhythmias, right or biventricular heart failure, to even sudden death, at any stage of the disease. From primary care consultations, we can capture asymptomatic patients suspected of having this disease. There are no clear guidelines regarding treatment of asymptomatic patients without arrhythmias. In patients with moderate risk, drug treatment is advisable. In patients with high arrhythmia risk, radiofrequency ablation, placement of an Implantable Cardioverter Defibrillator (ICD), and cardiac transplantation should be considered. There is evidence of the undeniable physical and psychological benefits that Cardiac Rehabilitation Programs achieve in ICD patients, as well as of the low risks that well-planned training poses.
\end{abstract}

Key words: Arrhythmogenic Right Ventricular Dysplasia. Treatment. Rehabilitation.

\section{INTRODUCCIÓN}

La Displasia o Miocardiopatía Arritmogénica del Ventrículo Derecho (MAVD) es una enfermedad del músculo cardíaco de origen genético, en la que el miocardio es sustituido por tejido fibroadiposo. Es una de las causas más comunes de muerte súbita (MS) en adultos jóvenes, siendo en algunas series del $20 \%{ }^{1}$. Presenta una agregación familiar hasta en el $50 \%$ de los casos con herencia autosómica dominante con expresión variable y penetrancia incompleta (30\%). También se ha descrito la herencia autosómica recesiva ${ }^{2}$. La prevalencia mundial se calcula entre 1/2.000 y 1/10.000 en la población adulta. En los pacientes afectados, puede progresar y afectar al ventrículo 
izquierdo, con una prevalencia de hasta el $76 \%$. La tasa anual de mortalidad anual sin tratamiento es de $2,5-3 \%$, y en individuos en tratamiento es del $1 \%{ }^{3}$.

La clínica es variable y va desde pacientes asintomáticos, arritmias ventriculares y supraventriculares, insuficiencia cardíaca derecha o biventricular, hasta muerte súbita, en cualquier fase de la enfermedad.

El electrocardiograma (ECG) puede ser inicialmente normal. Entre las alteraciones más frecuentes destacan la inversión de la onda T (V1-V3) en el $50 \%$ de los sujetos; bloqueo de rama derecha incompleto (18\%); prolongación del QRS en V1 y V2 y la aparición de la onda épsilon en aproximadamente el $30 \%$ de los pacientes, que puede no estar presente inicialmente y aparecer con posterioridad $^{4,5}$. El ecocardiograma es útil para el diagnóstico diferencial con otras patologías, y entre los hallazgos descritos se encuentran la dilatación e hipocinesia del ventrículo derecho, aneurismas telediastólicos y discinesia inferobasal. La prueba de esfuerzo y el Holter detectan arritmias que apoyan al diagnóstico ${ }^{1,4}$.

La resonancia nuclear magnética (RNM) cardiaca es una técnica no invasiva de alta sensibilidad, que orienta a la vista del resultado de otras pruebas complementarias $^{6}$. El diagnóstico definitivo requiere la confirmación mediante anatomía patológica de la sustitución fibroadiposa transmural pero, debido a la afectación segmentaria, a la complejidad de la técnica y sus complicaciones, no siempre se realiza.

\section{CASO CLÍNICO}

Varón de 22 años de edad que acude a la consulta de Atención primaria (AP) con antecedentes personales de soplo funcional en la infancia, rinitis alérgica estacional, fumador de 10 cigarrillos diarios y bebedor de fines de semana. Antecedentes familiares sin interés. Tras la realización de un ECG en la consulta de Atención Primaria requerido previo a viaje al extranjero para cursar estudios en una universidad privada del Reino Unido se objetiva una onda T negativa generalizada de V2 a V6 y en cara inferior, con extrasístoles ventriculares aislados (figura 1). En la exploración física presenta cifras tensionales de 140/70 mm Hg, auscultación cardiopulmonar normal a 60 latidos por minuto sin otros datos de interés. Con dichos hallazgos electrocardiográficos es derivado a la consulta de Cardiología.

Los estudios complementarios solicitados desde AP son normales (analítica con hemograma, bioquímica y coagulación; radiografía de tórax lateral y posteroanterior). El ecocardiograma detecta una dilatación importante del ventrículo derecho (55 $\mathrm{mm}$ ), con cara lateral hipoquinética así como una insuficiencia tricuspídea leve. El holter evidencia un ritmo sinusal y un marcapasos auricular migratorio con extrasistolia ventricular frecuente y algún triplete ventricular. La RNM cardíaca muestra un ventrículo derecho con aumento de tamaño e hipertrofia de banda moderadora con pared fina con algunas áreas hiperintensas en su interior sugerentes de infiltración grasa, con pequeñas saculaciones discinéticas. El ventrículo izquierdo, válvulas y pericardio son normales.

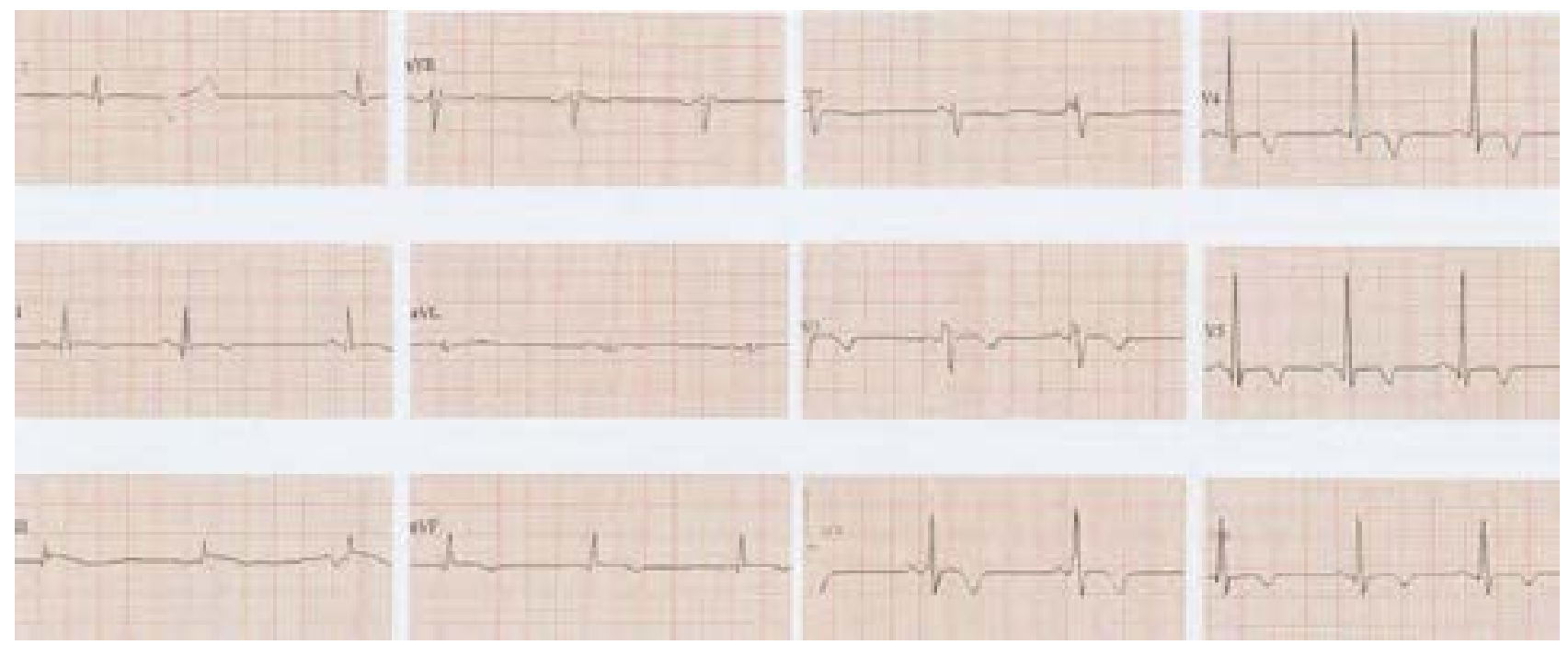

Figura 1. Electrocardiograma del paciente. Onda T negativa en V2-V6 con extrasístoles ventriculares aislados. 
Con la sospecha diagnóstica de MAVD se instaura tratamiento con betabloqueantes. El paciente permaneció asintomático siguiendo controles periódicos. Con posterioridad a su vuelta a España dos años después, tras jugar un partido de baloncesto, desarrolla un cuadro sincopal, por lo que finalmente se decide realizarle implantación de un DAI. Al mes del ingreso se remite al Servicio de Rehabilitación para valorar comenzar un Programa de Rehabilitación Cardiaca (PRC). El paciente sigue una vida activa en la actualidad, un año después, con seguimiento desde Atención Primaria y revisiones por Cardiología.

\section{DISCUSIÓN}

Esta entidad con riesgo de muerte súbita puede ser sospechada y detectada desde Atención Primaria, bien ante la presencia de clínica o ante pacientes asintomáticos en los que, tras realización de un ECG, aparezcan alteraciones compatibles a las descritas. El diagnóstico definitivo y tratamiento se instaura desde Atención Hospitalaria, pudiéndose realizar el seguimiento desde las consultas de Atención Primaria. Entre las medidas terapéuticas disponibles para el tratamiento de esta entidad se encuentran el tratamiento farmacológico con betabloqueantes y antiarrítmicos (amiodarona o sotalol), la ablación con radiofrecuencia, la implantación de DAl y el trasplante cardíaco ${ }^{3}$.

No existen directrices claras con respecto al tratamiento de pacientes asintomáticos y sin arritmias. La estratificación del riesgo cardiovascular (Tabla 1) está encaminada a prevenir la muerte súbita. En pacientes con riesgo moderado es aconsejable el tratamiento farmacológico con betabloqueantes y fármacos antiarrítmicos del grupo III. En los pacientes con riesgo elevado para desarrollar arritmias se debe considerar la ablación por radiofrecuencia, la colocación de un DAI7 y el trasplante cardiaco ${ }^{8}$.

En pacientes portadores de un desfibrilador cardioversor implantable (ICD), la actividad física recomendable tendrá las siguientes peculiaridades: no se recomienda el deporte de competición, salvo que el mismo implique baja demanda cardiovascular (golf, bolos, billar) y no presente riesgo de síncope; se deben evitar deportes de impacto y contacto, así como ejercicios extremos con el miembro superior ipsilateral y debe evitarse la exposición a campos magnéticos. Estarían recomendados los deportes recreacionales, a realizar a las 6 semanas del implante, y siempre atendiendo a la frecuencia cardíaca máxima permitida. Podemos supervisar desde Atención Primaria que estas directrices se cumplen.

Si existe activación del ICD, debemos remitir al paciente a Cardiología y recomendarle que la actividad física debe interrumpirse durante 6 semanas, así como en aquellas situaciones en las que sea necesario valorar un cambio en el tratamiento farmacológico o una reprogramación del ICD9.

El protocolo de entrenamiento de rehabilitación cardíaca recomendado a nuestro paciente, portador de un ICD es: ejercicio inicialmente supervisado en un centro hospitalario, comenzando tras seis semanas de la implantación del ICD durante tres meses, con una frecuencia de tres sesiones a la semana y con una duración de cada una de las sesiones de aproximadamente 90 minutos $^{9}$. La intensidad del ejercicio debe determinarse individualmente para cada paciente, y debería estar entre el 60 y el $90 \%$ de la frecuencia cardíaca obtenida en la fórmula de Karvonen (Frecuencia cardiaca de entrenamiento $\mathrm{FCE}=\mathrm{FC}$ basal $+\%$ [FC máxima-

\begin{tabular}{lccc}
\hline Riesgo & $\begin{array}{c}\text { Características clínicas } \\
\text { (Arritmias) }\end{array}$ & $\begin{array}{c}\text { Comportamiento de } \\
\text { la PAS durante el } \\
\text { ejercicio }\end{array}$ & $\begin{array}{c}\text { Tolerancia al ejercicio } \\
\text { durante la prueba de } \\
\text { esfuerzo }\end{array}$ \\
\hline Bajo & Ausencia & Normal & $\begin{array}{c}\text { Tolerancia máxima al ejercicio } \\
>7 \text { METS (equivalentes } \\
\text { metabólicos) }\end{array}$ \\
Medio & $\begin{array}{c}\text { Presencia de arritmias no } \\
\text { complejas ni frecuentes }\end{array}$ & Repuesta presora plana & $\begin{array}{c}\text { Tolerancia máxima al ejercicio } \\
5-7 \text { METS }\end{array}$ \\
Alto & $\begin{array}{c}\text { Presencia de arritmias } \\
\text { complejas o frecuentes. } \\
\text { Fibrilación auricular. }\end{array}$ & $\begin{array}{c}\text { Respuesta presora } \\
\text { hipotensiva }\end{array}$ & $\begin{array}{c}\text { Tolerancia máxima al ejercicio } \\
<5 \text { METS }\end{array}$ \\
\hline
\end{tabular}

Tabla 1. Estratificación del riesgo cardiovascular. Modificado de referencias 11-13. PAS: Presión arterial sistólica. 
FC basal]). Debe instruirse a los pacientes para no sobrepasar la frecuencia cardíaca indicada, para lo cual se debe monitorizar a todos. Si es preciso, el programa de rehabilitación debe complementarse con un programa de entrenamiento de la fuerza de los miembros superiores e inferiores y de la musculatura inspiratoria.

En ausencia de isquemia durante el ejercicio en la prueba de esfuerzo, el límite superior de la frecuencia cardíaca durante el ejercicio debe estar en valores de alrededor de 10-20 latidos por debajo de la frecuencia de descarga ${ }^{10}$.

Se le indicó a nuestro paciente desde la consulta evitar los ejercicios en decúbito prono, ya que pueden provocar úlceras por decúbito en la zona de implantación del DAl ${ }^{9}$.

En pacientes con MHVD portadores de DAl sería recomendable incluirlos en un tratamiento específico e individualizado por parte de los psicólogos y psiquiatras de la Unidad de Rehabilitación Cardíaca, con el fin de mejorar la calidad de vida y el pronóstico, ya que existe evidencia de la relación entre afectación psicológica, arritmias ventriculares y descargas del DAl ${ }^{9}$.

El apoyo del equipo de Atención Primaria y la accesibilidad también serán pilares importantes para una evolución adecuada del paciente y la reincorporación temprana a su vida cotidiana.

Existe evidencia de los indudables beneficios que a nivel físico y psicológico producen los PRC en portadores de DAl, y de los escasos riesgos del entrenamiento bien programado 9 .

Una vez realizado el PRC, tras el correcto aprendizaje del programa de entrenamiento y de las de pautas indicadas, es posible en estos pacientes conseguir una vida activa de calidad con seguimiento por parte de médico de Atención Primaria, en coordinación con Cardiología y Rehabilitación, mediante un circuito directo de derivación.

La tendencia actual es al establecimiento de PRC individualizados realizados en el domicilio y tutelados por los distintos profesionales sanitarios (médicos de AP y médicos del segundo nivel asistencial, enfermeras, psicólogos, trabajadores sociales) recibiendo material informativo, visitas, acceso a consulta o llamadas telefónicas que les resuelvan dudas y les incentiven a continuar con el ejercicio y los hábitos de vida cardiosaludable. Estos programas son especialmente útiles en pacientes que viven lejos del hospital.

\section{BIBLIOGRAFÍA}

1. Marcus FI, McKenna WJ, Sherrill D, Basso C, Bauce B, Bluemke DA et al. Diagnosis of arrhythmogenic right ventricular cardiomyopathy / dysplasia: proposed modification of the Task Force Criteria. Eur Heart J. 2010; 31 (7): 806-14.

2. Inciardi RM, Maresi E, Coppola G, Rotolo A, Clemenza F, Giordano $U$ et al. Anatomical features and clinical correlations in Caucasian patients with definite arrhythmogenic right ventricular dysplasia / cardiomyopathy. Minerva Cardioangiol. 2014; 62 (5): 369-78.

3. Retyk EO, director. Consenso de prevención primaria y secundaria de muerte súbita. Sociedad Argentina de Cardiología - Sociedad Uruguaya de Cardiología (con la colaboración del CONAREC). Rev Argent Cardiol. 2012; 80 (2): 165-84.

4. Quarta G, Elliott PM. Criterios diagnósticos para la miocardiopatía arritmogénica de ventrículo derecho. Rev Esp Cardiol. 2012; 65 (7): 599-605.

5. Benito B, Brugada J, Brugada R, Brugada P. Síndrome de Brugada. Rev Esp Cardiol. 2009; 62 (11): 1297-315.

6. Nucifora G, Muser D, Masci PG, Barison A, Rebellato L, Piccoli $G$ et al. Prevalence and prognostic value of concealed structural abnormalities in patients with apparently idiopathic ventricular arrhythmias of left versus right ventricular origin: a magnetic resonance imaging study. Circ Arrhythm Electrophysiol. 2014; 7 (3): 456-62.

7. Schinkel AF. Implantable cardioverter defibrillators in arrhythmogenic right ventricular dysplasia / cardiomyopathy: patient outcomes, incidence of appropriate and inappropriate interventions, and complications. Circ Arrhythm Electrophysiol. 2013; Jun; 6 (3):562-8.

8. Cruz Cardentey M, Méndez Rosabal A. Displasia arritmogénica de ventrículo derecho. Rev Cubana Cardiol Cir Cardiovasc. 2011; 17 (2): 159-66.

9. Galindo Nogueras E, Pleguezuelos Cobo E, Pérez Mesquina ME. Arritmias, ejercicio y rehabilitación. En: Pleguezuelos Cobo E, Miranda Calderón G, Gómez González A, Capella Sans L, edit. Principios de Rehabilitación Cardíaca. Madrid: Editorial Médica Panamericana. 2011. p. 224-5.

10. Vanhees L, Kornaat M, Defoor J, Aufdemkampe G, Schepers $D$, Stevens $A$ et al. Effect of exercise training in patients with an implantable cardioverter defibrillator. Eur Heart J. 2004; 25 (13): 1120-6.

11. Castillo JI, Moreno Atanasio J, Tonoco González J y Pleguezuelos Cobo E. Rehabilitación cardiaca en el síndrome coronario agudo. En: Pleguezuelos Cobo E, Miranda Calderón G, Gómez González A, Capella Sans L, edit. Principios de Rehabilitación Cardíaca. Madrid: Editorial Médica Panamericana. 2011. p. 169-81.

12. Myers J, Prakash M, Froelicher V, Do D, Partington S, Atwood E. Exercise capacity and mortality among men referred for exercise testing. N Engl Med. 2002; 346 (11): 793-801.

13. López-Jiménez F, Pérez-Terzic $C$, Zeballos PC, Anchique CV, Burdiat G, González K et al. Consenso de rehabilitación cardiovascular y prevención secundaria de las Sociedades Interamericana y Sudamericana de Cardiología. Rev Urug Cardiol. 2013; 28 (2): 189-224. 